\title{
Reflexiones sobre el poder y el ejercicio de la autonomía en los espacios de participación incorporados a la gestión de la política pública*
}

\section{Reflections on the power and the exercise of the autonomy in participative spaces instituted in the management of public policies}

\author{
María del Socorro Foio **
}

\section{Resumen}

La intención de este artículo ha sido aportar a los modos posibles de realizar lecturas sobre los espacios participativos instituidos en la gestión de políticas públicas.

En la literatura consultada hemos advertido dos grandes tendencias en la interpretación de los espacios de participación: una que permite pensar que estos construyen formas de tomar decisiones vinculadas al orden democrático en tanto favorecen el ejercicio de autonomías relativas de los actores sociales, y otra ligada a entender la gestión pública en el marco de la construcción de un proyecto heterónomo y burocrático.

Ello supone en las políticas públicas la existencia de la tensión entre una gestión que pueda contemplar lo contextualmente situado (la singularidad, podemos decir, y que entonces refiere a una concepción democrática abierta a la construcción de nuevos significados), y una gestión que no lo contempla (y es por tanto autoritaria como concepción al imponer los significados de manera heterónoma).

\footnotetext{
* Artículo recibido el 28 de Septiembre de 2015. Aceptado el 27 de Diciembre de 2015.

** Universidad Nacional del Nordeste. Argentina. Correo electrónico: socorrofoio@gmail.com.
} 


\section{Abstract}

The aim of the article has been to contribute to the possible ways of doing readings on the participative spaces instituted in the management of public policies.

In the literature we have consulted, we have noticed two important trends in the interpretation of participative spaces: on the one hand the trend that suggests that these spaces build ways to take decisions related to the democratic order as they favour the exercise of relative autonomies of the social actors; and on the other hand the trend linked to understand the public management in the context of building an heteronomous and bureaucratic project.

In the public policies it supposes the existence of tension between management that can contemplate what is contextually located (the singularity, we can say, and then refers to a democratic conception open to the construction of new meanings), and a management that does not include it (and is therefore authoritative as conception when it imposes the meanings in an heteronomous way).

\section{Palabras clave}

Sentidos de la participación - Ámbitos de encuentro y negociación - Construcción de ciudadanía.

\section{Keywords}

Senses of the participation - Areas of meeting and negotiation Construction of citizenship.

\section{Sobre el objeto de análisis}

En este artículo abordamos una forma contemporánea de manifestación de la política, la gestión de la política pública, y la situamos en la región de América Latina y el Caribe. En ese contexto, el objeto material de análisis son las prácticas participativas promovidas por el Estado en el diseño y ejecución de políticas públicas.

Al haberla definido como el conjunto de decisiones adoptadas en un terreno donde el poder es constitutivo, la política es considerada por Laclau como una ontología de lo social ya que "lo social solo puede consistir en las formas sedimentadas de un poder que ha borrado las huellas de su propia contingencia" (Laclau, 1994: 23). Desde esta perspectiva, entonces, la política se concibe como una actividad que debe lidiar con la heterogeneidad o la alteridad, con la circunstancia de que las estructuras de la sociedad 
no tienen un fundamento más allá de la contingencia del estar juntos o de ser consecuencia del hecho de que no son dictadas por el potencial genético.

En el marco en que se desarrolla nuestro análisis entendemos la política en tanto actividad innegociable que, partiendo de lo individual, se expresa en la conciencia de la necesidad de participar en mutualidad en la creación de mejores condiciones para todos y se realiza mediante discursos y acciones colectivas.

Esa acción colectiva, según Alvarado Salgado está orientada, fundamentalmente, a incidir sobre las creencias, relaciones y prácticas sociales que afectan al bienestar común, cuestionando y cambiando los ejercicios de poder que se dan en ellas (Alvarado Salgado, 2013).

Ante la necesidad de reducir la complejidad que asume la tensión Estado democrático-capitalismo, los gobiernos, actuando directamente o a través de agentes, realizan un conjunto planificado de actividades específicas de transformación y combinación de recursos normativos, financieros y tecnológicos que se configura como política pública. Se trata de políticas que buscan tener una influencia determinada sobre la vida de los ciudadanos (atender problemas, controlar comportamientos, satisfacer demandas) con el fin de lograr impactos sociales, políticos y económicos (Subirats, 1989; Ballart, 1992; Repetto, 2001; Piñango, 2003).

Como dijimos, nuestra mirada se ha dirigido hacia políticas públicas que en sus objetivos formales prevén la habilitación de instancias participativas en la gestión de estas como medio específico referido a la finalidad explícita de crear oportunidades y fortalecer instrumentos para la equidad y la integración social, a través del mejoramiento de la capacidad de intervención de los destinatarios, en tanto actores autónomos, en las esferas políticas y estatales.

En tal sentido, hemos explorado diversas ideas y conceptos vinculados con esta problemática, con la finalidad de analizar las condiciones de posibilidad de los espacios que resultan de la convocatoria del Estado a la participación de los actores involucrados durante la gestión de la política pública en países de la región.

Es decir, nuestro objetivo es alcanzar a comprender en qué medida dichos espacios operan como dispositivos donde el ejercicio de la democracia y la autonomía individual y colectiva se produce efectivamente, o tienden a ser ámbitos donde la toma de decisión funciona guiada por una concepción burocrática y heterónoma de la política.

Nuestras reflexiones reconocen entre sus fuentes de inspiración algunos análisis preliminares que hemos realizado sobre un conjunto seleccionado de experiencias institucionalizadas de participación en el diseño, ejecución y/o evaluación de la política pública respectiva, desarrolladas en doce países de América Latina y el Caribe. Las políticas consideradas en esta ocasión son las siguientes: en Argentina, Programa Jefes y Jefas de Hogar Desocupados (2002-2004), Política de Vivienda de la provincia de Córdoba (1992-1999), y Programa de Transición al Gobierno por Comunas de la Ciudad Autónoma de Buenos Aires (2006-2007); en Bolivia, Planes de Desarrollo Municipal, 
Política de Descentralización, Ley de Participación Popular (1994-2005); en Brasil, Planes Directores Urbano Ambientales en Ciudades Brasileñas (2004-2005); en Chile, Programa Puente, Observatorio Social de la Participación (2002-2005); en Colombia, Red de Solidaridad Social (1994-1998); en Cuba, Programa de Transformación integral del Barrio Jesús María (2008-2009); en Ecuador, Política Nacional de Salud (2003-2004); en Guatemala, Programa Guate Solidaria Rural (2004-2008); en México, Programa Oportunidades (2001-2006); en Paraguay, Programa Reforma Educativa Escuela Viva (2001-2006); en Perú, Política de Juventud (2003-2010); en Uruguay, Cartera de Inmuebles para Vivienda de Interés Social (2009-2013); en Venezuela, Programa de Creación de los Consejos Comunales (2006 en adelante).

Las referencias de las mencionadas políticas las constituyen diversos documentos publicados en la región ${ }^{1}$, donde se reseñan las experiencias arriba mencionadas. Dichas reseñas constituyen comunicaciones de contenido que permiten explicitar -a través del discurso que se produce en ámbitos académicos y en organizaciones civiles de investigación y desarrollo-, qué referencias creíbles otorga la sociedad (Verón, 1969; Pintos, 1994) acerca de cómo pensar la participación en tanto realidad comprobable de la gestión de las políticas públicas en países de América Latina y el Caribe.

\section{Una introducción acerca de las políticas sociales y la participación ciudadana}

En el campo de las políticas públicas, según la perspectiva aquí planteada, la función principal de la política social es la reducción y eliminación de las inequidades sociales a través de la redistribución de los recursos, servicios, oportunidades y capacidades. En sus objetivos manifiestos, el Estado apela a esta política para construir una sociedad integrada y equitativa, con el fin de facilitar la convergencia entre los intereses individuales y los intereses comunes.

Las políticas sociales y sus expresiones programáticas comprenden intervenciones sectoriales clásicas (educación, salud, seguridad social, vivienda, infraestructura básica)

\footnotetext{
${ }^{1}$ Observatorio Social (2006) Cartillas de Observación, Documentos académicos, Buenos Aires: Programa para el análisis de procesos participativos de diseño e implementación de políticas sociales (en soporte digital); Heras, A., Córdova, L. y Burin, D. (2007) Análisis de la participación en la transición hacia el gobierno por comunas de la ciudad de Buenos Aires, Estudios Políticos, 31, Instituto de Estudios Políticos, Medellín: Universidad de Antioquia, pp.183-229; Ortega González, D. (2012) Estado centralista. Fortalezas y limitaciones de la experiencia cubana, en Pérez Rubio, A. y Antequera Durán, N., ed. (2012) Viejos problemas, nuevas alternativas. Estrategias de lucha contra la pobreza gestadas desde el Sur, Buenos Aires: CLACSO; pp.305-329; Arévalo, M. (2012) Programas sociales asistencialistas en Guatemala, 2004-2008, en Pérez Rubio, A. y Antequera Durán, N., ed. (2012) Viejos problemas, nuevas alternativas. Estrategias de lucha contra la pobreza gestadas desde el Sur, Buenos Aires: CLACSO, pp.23-47; Rodríguez González, N. (2013) Sentidos en torno a la participación en las cooperativas de vivienda por ayuda mutua en formación. Primera aproximación al campo de problemas, Revista Latinoamericana de Psicología Social IMB - Número 2 - Santiago de Chile: Universidad Alberto Hurtado, pp.137-157; Ellner, S. (2012) El modelo de la democracia social radical en Venezuela: innovaciones y limitaciones. Cuadernos del CENDES, Año 29, $N^{\circ} 79$, Tercera época, Caracas: Universidad Central de Venezuela, pp.107-133.
} 
así como las tendencias a desarrollar acciones focalizadas en la pobreza, a la vez que también deben incorporarse bajo el concepto de política social las intervenciones estatales destinadas a promover el empleo y brindar protección ante los males sociales (Repetto, 2005).

En los procesos de diseño y adopción de planes y programas a llevar a cabo durante la gestión de las políticas sociales, la inclusión de la ciudadanía con reglas de actuación de los diferentes actores previamente definidas aparece como particularmente necesaria para proporcionar eficacia a una acción del Estado que suele reducirse al ámbito gubernamental y no de lo público.

Esta problemática es especialmente considerada por la agenda actual de la ciencia social al contemplarse en ella el desarrollo de conceptos e ideas en torno a cómo y por qué impulsar la participación ciudadana en las decisiones públicas, incorporando sus intereses sobre determinadas temáticas. La idea central es que la participación permite avanzar en el camino de construir ciudadanía.

Al vincular al Estado y la sociedad civil dentro de una cultura política que orienta el comportamiento de los sujetos individuales y colectivos en un marco de deberes y derechos ciudadanos, la participación contribuye a consolidar el sistema democrático y asegurar su sustentabilidad al otorgarle mayor legitimidad social (Cunill, 1991).

Sin embargo, cuando se trata de políticas sociales transitar hacia la corresponsabilidad entre gobierno y ciudadanía es algo complejo y que no está bien resuelto en la acción pública social desarrollada en América Latina y el Caribe, en parte debido a que las nuevas iniciativas o criterios de gestión de políticas sociales no operan en el vacío, sino en contextos dados con instituciones y prácticas heredadas (Ziccardi, 2012).

Sin duda, el éxito de la participación en las políticas sociales depende del logro de una combinación de compromisos y movilización de los actores sociales involucrados y de la capacidad de las instituciones para impulsar innovaciones que aseguren reglas claras de representación política, de los procesos de discusión y de la toma de decisiones, capaces de garantizar una participación efectiva de quienes tienen menos recursos argumentativos y conocimientos técnicos. En los proyectos que deriven de la política social, la promoción de estrategias que fortalezcan prácticas asociativas de las ciudadanas y ciudadanos no organizados podrá contribuir a potenciar un cambio hacia una cultura más participativa.

\section{El contexto y la contingencia histórica en el que se desarrollan las políticas públicas con sesgo participativo}

El aspecto más característico de la región de América Latina y el Caribe es la condición de ser el continente de mayor desigualdad de ingresos, de género y de etnia del mundo, resultante de la heterogeneidad estructural.

Se trata de sociedades de tipo dual, en las que se constituyen circuitos altamente diferenciados para ricos y pobres y donde la eliminación de la pobreza no es suficiente 
para reducir la desigualdad, ya que las oportunidades de interacción son bajas y suceden en contextos que profundizan la percepción como distintos. De ahí que, en algunos casos, los miembros de las instancias participativas tengan características de élite y con ello se discuta si esas instancias atenúan o acrecientan la desigualdad.

Los distintos momentos en que se desarrollan las políticas consideradas en este artículo reconocen como antecedente la etapa de imposición durante la década de 1970 de dictaduras cívico-militares y terrorismo de Estado, alentados por Estados Unidos en el marco de la guerra fría. Varios de los países a los que pertenecen las experiencias estudiadas sufrieron esas dictaduras; a fines de aquella década, en la región solo Cuba -cuyo sistema político es afín a su trayectoria revolucionaria-, Colombia, Costa Rica, México y Venezuela no presentaban dictaduras de aquel tipo.

Una década después, en un escenario mundial de hegemonía neoliberal, se fueron reconstituyendo los regímenes democráticos, en un proceso de transición que reconoce diferentes grados de condicionamiento y en el que las amenazas antidemocráticas aparecen enmascaradas en actos desestabilizadores (Rivas, 2013). Casos de transiciones más condicionadas son Paraguay y Chile. Los condicionamientos fueron de menor grado en Brasil, Argentina y Uruguay, pero igualmente resultaron considerables y subsisten en cierta medida. Ecuador y Bolivia disponen de gobiernos que promueven medidas de mayor confrontación con los poderes fácticos. En Venezuela el proceso político impulsa un peculiar socialismo, en el marco de la llamada democracia participativa y protagónica (Rivas, 2013).

El nuevo liberalismo impuesto a escala global trajo para el continente un modelo de ajuste impuesto por el Consenso de Washington, que implicó la desprotección laboral y la desocupación creciente a la par del agotamiento en las estructuras de mediación partidos políticos y sindicatos- provocados por el aumento de la conflictividad del capitalismo tardío a partir de las modificaciones tecnológicas del proceso productivo y las modalidades de valorización del capital (Borón, 1993).

Las políticas públicas acentuaron aspectos tales como la educabilidad y el aumento de la empleabilidad en tanto elementos clave que contribuirían al empoderamiento de quienes pertenecen a sectores vulnerables, ya que -según la lógica económica entonces vigente- esas personas serían responsables de su situación al no reunir las cualidades necesarias para participar competitivamente de la sociedad/mercado (Pérez Rubio y Antequera Durán, 2012).

De hecho, las políticas neoliberales desarrolladas en este período provocaron lo que Álvarez Leguizamón (2009) denomina una mutación discursiva y práctica de las políticas, los derechos sociales y laborales generando políticas llamadas, paradójicamente, de desarrollo humano que sirvieron para reproducir y profundizar aún más la pobreza y la desigualdad.

En esta estructura social caracterizada por la exclusión, se produce la irrupción de nuevos actores sociales que confrontaron activamente con la nueva situación, pero sus 
estrategias de resistencia resultaron mecanismos de sobrevivencia poco eficaces para cambiar en el corto plazo las condiciones del contexto (Feijoó, 2006).

Orientada a combatir ineficiencias del Estado en la resolución de problemas que afectan a las comunidades, se promoverá la participación ciudadana -a instancias de las agencias multinacionales de crédito- con el propósito de ampliar la influencia de la sociedad civil sobre el proceso de toma de decisiones en todos los niveles, escalas y sectores de la gestión pública.

En ese marco, los gobiernos de la región propulsaron la apertura de espacios participativos en la gestión de las políticas públicas.

En términos generales, la creación de dichos espacios mediante mecanismos, procesos y estructuras institucionalizados por una normativa legal, organizaría la intervención de los ciudadanos en acciones gubernamentales, en tanto sujetos individuales o colectivos portadores de intereses reconocidos como públicos y políticos.

Su propósito explícito apuntaba a garantizar condiciones de igualación para el intercambio entre actores con distinto poder material y simbólico, impulsar cambios significativos que elevasen la calidad institucional en los procesos de toma de decisiones, o permitir el acceso a mejores condiciones de vida de los ciudadanos.

El impacto de las medidas neoliberales consagradas en la década de 1990 unido a la desvinculación entre dirigidos y dirigentes en los partidos políticos y organizaciones sindicales generó profundas reacciones y cuestionamientos que en la proximidad del nuevo siglo darían lugar a la emergencia de otras realidades en la acción política (Heras Monner Sans, 2011). En esta nueva etapa se instituyeron en la región políticas de derechos humanos para terminar con la impunidad, estrechando relaciones interamericanas en organizaciones que incluyen la defensa de las instituciones democráticas (Rivas, 2013) y, en lo que respecta a la gestión estatal, varios países emprendieron un camino alternativo al modo económico-político dominante, mediante experiencias de gobierno posneoliberales, condicionadas por la crisis del capitalismo financiarizado global.

Esto conlleva un proceso de reformas destinado a ensanchar el mercado interno, disminuir la desocupación y propiciar el acceso al consumo a sectores postergados, con una matriz redistribucionista, así como el despliegue de una integración ético-política entre los Estados orientada a encarar los asuntos regionales desde ámbitos progresivamente autónomos de la hegemonía norteamericana -ALBA, Petrocaribe, UNASUR- que desempeñan un papel clave en la expansión y afianzamiento de la legitimidad de los gobiernos democráticos (Foio, 2014).

Así, el posicionamiento de la región se fue perfilando en rechazo a las doctrinas de libre comercio desregulacionista y en la certeza de constituir una masa crítica que permita gravitar políticamente en el escenario global sin subordinarse a las agencias que pretenden controlar a las naciones en base a los principios neoliberales (Toer et al., 
2011). Esa búsqueda de mayores niveles de autonomía ha implicado tener que procurar recursos para la intervención estatal en la reactivación económica, medida que se enlaza con el incentivo de la politización popular expresada en instancias de movilización y participación ciudadanas. ${ }^{2}$

En ese nuevo escenario, según Sader (2012), las políticas de desarrollo social que unen expansión económica con distribución de renta impulsadas por los Estados como reacción a las medidas neoliberales consagradas en la década de 1990 convirtieron a América Latina y el Caribe en el continente que más ha disminuido la desigualdad en el período 2002-2012.

No obstante ello, las dificultades que enfrentan nuestros países son importantes debido a que se parte de un Estado desarticulado, de una sociedad fragmentada. $Y$, a su vez, el contexto internacional sigue siendo predominantemente neoliberal, lo cual significa recesión y financiarización de la economía mundial, en un marco exportador latinoamericano cada vez más primario.

Así, experiencias de gobierno que han desmercantilizado la acción estatal al privilegiar derechos como la educación, la salud, el transporte, retomando las prácticas de un Estado social constructor de nuevas formas de sociabilidad, de humanismo, de solidaridad -valores contrapuestos a los que privilegia la economía de mercado-, encuentran un fuerte obstáculo en la desafiliación y la marginalidad resultantes del orden neoliberal y que siguen manteniéndose en la actualidad (Sader, 2012).

Entendemos que es por esta razón que las modalidades participativas promovidas desde el sector gubernamental no alcanzan a revertir los conflictos distributivos que demandan cambios políticos, sociales e institucionales.

Con referencia a esta situación, Amadeo y Caputo advierten acerca de la configuración de "un sistema de reglas que restringe o acrecienta las posibilidades para la integración social" (Amadeo y Caputo, 2006: 20) resultante de las confrontaciones entre las condiciones para lograr una mejora distributiva en la sociedad y las estructuras y relaciones de poder y legitimación social de los actores. Por su parte Fleury plantea la necesidad de una reflexión sobre cómo puede evitar el Estado en la mediación de los conflictos distributivos "correr el riesgo de disciplinar o cooptar el movimiento molecular que densifica la esfera pública" (Fleury, 2008: 23).

\section{Interpretaciones de la tensión autonomía-heteronomía en la política pública con sesgo participativo}

\footnotetext{
2 Para Toer, en este contexto político, las estrategias de las fuerzas de oposición se relacionan con la defensa de intereses sectoriales y económico-corporativos y la apropiación de temas de las agendas posglobalización -ecología, indigenismo-, valiéndose, en gran medida, para el desarrollo de esas estrategias de los medios informativos como instrumento organizador del discurso propio y de apelación a los sectores subalternos (Toer et al., 2011).
} 
En la literatura sobre el tema, podemos encontrar dos grandes tendencias en la interpretación de los espacios participativos.

Una de ellas es la que permite pensarlos como ámbitos que construyen formas de tomar decisiones vinculadas al orden democrático en tanto favorecen el ejercicio de autonomías relativas de los actores sociales.

La otra mirada aparece ligada a formas de gestión pública que se vinculan con el orden heterónomo y burocrático de entender la política.

Entendemos que estas corrientes contrapuestas, actualmente en vigencia, responden a distintas visiones de la política.

Existe, así, una línea que percibe a la política como instancia de lucha de intereses enfrentados, expresión de una voluntad de dominio explícita o implícita, que se vale de tecnologías de sometimiento. Agotado el espacio público y cimentada una clase política distante de la ciudadanía, la instrumentalización del poder se convierte en una vía eficaz para el logro de los objetivos en disputa, en un orden heterónomo que coarta las posibilidades de deliberación democrática.

La otra concepción, partiendo de la misma definición de situación, ha propiciado la reflexión crítica sobre el poder a través del análisis de las instituciones y técnicas que los agentes reales construyen en su quehacer y sus relaciones con otros agentes. Es un enfoque que visualiza las relaciones de poder que producen desigualdades, reconociendo que al mismo tiempo son condiciones de estas, y revela los puntos de resistencia presentes en la red de poder de donde podrían emerger transformaciones radicales cuyo destino es la autonomía (Castoriadis, 2006; López Yáñez, 2003).

Esta perspectiva de análisis también hace visible que existen cambios que posibilitan a las personas relacionarse a través de la palabra en asuntos de interés común, por una parte, y, a su vez, posibilita identificar, describir y analizar formas de asumir iniciativas, de generar algo nuevo en el espacio público y de ver entonces a dicho espacio como lugar de desenvolvimiento de la política, como fin en sí mismo.

Esta dimensión espacial permite proponer que en estos espacios se produce desde la pluralidad, al coordinar diversas posibilidades de percepción y de acción entre aquellos que ocupan posiciones distintas (García Raggio, 2008).

\section{Sentidos de la participación en los espacios promovidos en la gestión de políticas públicas}

Con el fin de las dictaduras cívico-militares en la región, la paulatina democratización de las instituciones del Estado tuvo su influencia en el movimiento orientado a la planificación estratégica y la participación de la sociedad civil en la gestión de las políticas públicas.

Proyectos de desarrollo local, desarrollo territorial rural, gobernanza ambiental descentralizada, entre otros, fueron diseñados como respuesta a las movilizaciones y conflictos distributivos con el propósito de ejercer un rol estimulador del crecimiento 
de la comunidad mediante la promoción de espacios multiactorales de participación social (Foio, 2007).

Sin embargo, en ciertas oportunidades la manipulación política de estos procesos que, en su momento, fueron pensados como pluralistas y democratizadores- los habría vaciado de contenido participativo e incluyente (Heras y Foio, 2007).

Amadeo y Caputo señalan con respecto a muchas de esas propuestas, que ellas: (constituyen) argumentos funcionales según los cuales la participación sería un mecanismo pertinente para superar los obstáculos que suelen encontrarse en la resolución de los problemas cuando estas acciones se llevan adelante sin tener en cuenta la voz y necesidades de los propios interesados (Amadeo y Caputo, 2006: 4).

Por nuestra parte, en la intervención en diferentes estudios realizados en Argentina, pudimos notar que ciertas prácticas participativas promovidas por el Estado tienden a naturalizar la desigualdad en la distribución de la riqueza y los recursos. ${ }^{3}$

Específicamente, en las comunicaciones de contenido de las experiencias a las que refiere el presente trabajo hemos observado los procedimientos por los que los diferentes emisores transmiten los rasgos característicos de su realidad que pretende ser presentada como la realidad de la participación.

Ello nos llevó a advertir la existencia de variaciones en los sentidos de la participación, que abarcan las referencias de percepción, explicación e intervención de los actores en los espacios instituidos. Y lo que consideramos como interesante es que, en la totalidad de las experiencias, las variaciones observadas ocurren, con distintas densidades, durante la gestión de un mismo proyecto en un tiempo no fijo, dando cuenta de la recursividad de los procesos que se desarrollan en el universo complejo de la política pública con componente participativo.

En los discursos analizados, hemos identificado los campos semánticos, o criterios, en los que cada acontecimiento comunicado cobra sentido según su grado de visibilidad social y posibilidad de conformación como suceso reproductor de las situaciones de heteronomía o como propulsor de las acciones autónomas.

Esos criterios son: el control (donde se hace más visible la ausencia de condiciones para la emergencia de proyectos de autonomía), la cooptación (mediante prácticas que vuelven visible las situaciones de heteronomía), la concertación (que emerge de procesos menos visibles de adaptación al conflicto heteronomía-autonomía) y la democratización (dando lugar a situaciones no tan visibles pero que preanuncian la emergencia de proyectos de autonomía).

En tanto campos de sentido, los criterios de selección de los acontecimientos significativos presentes en el discurso sobre la participación se sitúan en relación con el horizonte de comprensión, o entorno, fijado por las representaciones de los actores

\footnotetext{
${ }^{3}$ Pérez Rubio y Foio, 2009; Foio y Pérez Rubio, 2009, 2011, 2012 a, 2012 b; Foio, 2009; Almirón et al., 2006.
} 
sociales, las instituciones y las disposiciones de los sujetos o de su actividad que se ponen en juego en el espacio participativo.

Así, pudimos observar que, en el campo semántico del control, adquieren sentido las comunicaciones que recrean en la relación gobierno-beneficiarios condiciones de asimetría en el ejercicio de la autoridad y el manejo de recursos; estas refieren a: organismos gubernamentales, reproducción de mecanismos de representación, caudillismo, clientelismo, desconfianza, ocultamiento de la información, discontinuidad de la política y desarticulación institucional.

A su vez, en el campo semántico de la cooptación, adquieren sentido aquellas comunicaciones que suponen un reemplazo de la matriz clientelar asociada al despliegue de las estrategias de control, poniendo de manifiesto la debilidad de los actores subalternos - dirigentes populares, organizaciones o familias y personas no organizadas - para imponer sus intereses así como la posibilidad de los movimientos sociales de volver al orden al dejar de lado la ambición por la autonomía con respecto al gobierno. Esas comunicaciones refieren a: equipos técnicos, beneficiarios no organizados, bajo nivel de institucionalidad pública, concentración de poder, restricción de intervenciones y consultas, crecimiento asimétrico de la dirigencia, movilizaciones impulsadas por intereses del gobierno y participación como eslogan.

En cuanto al campo semántico de la concertación, alcanzan su sentido aquellas comunicaciones que remiten a actores poseedores de una capacidad de negociación para incidir en la gestión de la política pública a partir de la movilización de recursos propios, tienen acceso a la información relevante para poder confrontar con otras posiciones e influenciar en las diferentes instancias y cuentan con la legitimidad y/o legalidad necesarias para actuar en nombre de otros, interviniendo en el espacio de participación con miras a producir alianzas o sinergias que permitan la gobernabilidad. Se trata de comunicaciones que refieren a encuentros multiactorales, consultores y expertos, disputa y negociación, análisis de escenarios y coyuntura, gestión asociada, coordinación territorial e institucional, capacitación y capital humano y social.

Por su parte, al localizar en el espacio participativo el campo semántico de la democratización, logran sentido las comunicaciones alusivas a la búsqueda de condiciones de igualdad, al fortalecimiento de proyectos más que de acciones esporádicas y a la capacidad de los sujetos de asociarse y organizarse de tal modo que puedan ejercer una influencia directa en las decisiones públicas, habilitando instancias de deliberación que hacen hincapié en el respeto a las minorías, a las personas y colectividades. Son comunicaciones que refieren a: movimientos sociales y grupos de base, actores con voluntad de incidencia en lo público, deliberación, flexibilidad institucional, interculturalidad, formación política, asociatividad y vínculos de paridad aproximada.

El desarrollo de procesos democráticos a nivel de los grupos de base da cuenta de una participación entendida como proceso de empoderamiento y experiencia 
educativa, cuyo objetivo es promover el crecimiento y la equidad. Tal lo sostenido por aquellas propuestas teóricas que consideran fundamental la intervención de los destinatarios en la formulación de las políticas sociales, el diseño de los programas y su ejecución en niveles locales, compartiendo el acceso a los beneficios de dichos programas (Spicker, Álvarez Leguizamón y Gordon, 2009).

Como pudimos advertir, existen sentidos en el espacio participativo que aluden al surgimiento de nuevas formas de manifestación de la política con voluntad de transformación. Esa perspectiva transformadora se llegará a manifestar en aquellas situaciones en las que los sectores populares tienen mayor incidencia, al poder conformar de esa manera un ámbito común donde es posible definir y consensuar visiones y estrategias propias.

Amadeo y Caputo afirman que la institucionalización de ámbitos de participación llegará a constituir "un elemento decisivo para lograr mayores niveles de inclusión e integración social" (Amadeo y Caputo, 2006: 28) si de esos espacios resultan cambios que favorezcan tanto la ampliación de las posibilidades de acceso a bienes y servicios, como el aumento en los grados de libertad -conocimientos, habilidades, opciones- de las personas para elegir de qué manera quieren vivir.

Con respecto a la capacidad de los espacios de participación en la emergencia de proyectos de autonomía, Matellanes (1999) sostiene que la inclusión activa de los sectores populares en esos espacios abona la irrupción de un sistema de pensamiento y coordinación de acciones que cuestionará la capacidad del orden hegemónico para garantizar la igualdad social y llevará a plantear alternativas que favorezcan de manera efectiva la inclusión y la construcción de ciudadanía.

En una línea similar, Repetto (2001) destaca el potencial de movilización social y de acción colectiva que generan las estrategias participativas, tanto como medio de presión para el efectivo cumplimiento en la gestión de la política pública como para crear y autogestionar nuevas opciones por fuera de ella.

\section{Ejercicio de la ciudadanía y práctica democrática en los espacios participativos}

Según Luhmann (2006), el problema del orden social se manifiesta en una tensión que configura y posiciona al sujeto en uno u otro lado de la diferencia inclusión/exclusión, es decir, desde posiciones heterónomas, desarticuladas que se sostienen en la naturalización del orden social, hacia posiciones más críticas, autónomas, organizadas y contra-hegemónicas.

Desde esta perspectiva, la posibilidad de que se dé tal transición estará asociada con prácticas de participación genuina.

Las relaciones de poder que se manifiestan en toda organización producen -por sus materialidades y por sus significados, y a través de las comunicaciones que las sustentan- estructuras de poder. 
Siguiendo a Guattari, Heras Monner Sans (2011) refiere a transformaciones permanentes micropolíticas, moleculares, resultado de los deseos que resisten a reducirse en una unidad ideal que represente y mediatice la multiplicidad de intereses.

López Yañez (2005) menciona la puesta en juego de intereses de los grupos y las negociaciones con los intereses y objetivos de la organización como el marco micropolítico que muestra al poder como proceso instituyente en el cual los participantes en la comunicación pueden utilizar diferentes bases para configurar su posición de poder.

La cuestión de cómo incorporar activamente a la ciudadanía en las decisiones y acciones gubernamentales forma parte de los contenidos discursivos de las políticas públicas y de la legislación en la que se sustentan.

Pero no siempre se vislumbran coincidencias entre los preceptos sobre la participación de la ciudadanía definidos en las políticas y las actividades efectivamente desarrolladas en los planes y programas.

Así, en algunos de aquellos, los ciudadanos son concebidos e incorporados exclusivamente como beneficiarios $y$, si las circunstancias lo exigieran, podrían improvisarse formas e instrumentos de participación.

Formas que por lo general serían poco incluyentes en el sentido de considerar la desigualdad social e instrumentos que no mostrarían efectividad para transformar los procesos decisorios para hacerlos más eficaces y democráticos.

Al referirse a la actividad de los espacios de participación ciudadana, Cunill considera que tal participación puede ser condicionada y hasta neutralizada bajo una aparente práctica democrática, y constituirse "en una plataforma de fácil uso para construir artificialmente consensos y legitimar desigualdades" (Cunill, 1991: 9).

En esos casos, las actividades tienden a reducirse a la consulta, no siempre vinculante, para las decisiones y para la implementación de las acciones específicas de los programas.

$Y$, en las situaciones en las que los mecanismos de participación alcanzan el nivel de las decisiones, ello no necesariamente significa que se ejecute lo acordado, de hecho, el grado de concertación previa que exista entre todos los actores intervinientes es condición para poder llevar a cabo los acuerdos que se establezcan en los espacios participativos.

El principal cuestionamiento a los espacios participativos implementados durante la gestión de políticas públicas en la región, surge al interpretarlos como intervenciones generadoras y moduladoras de la desigualdad, que naturalizan o institucionalizan discriminaciones de clase, género, edad, etnia, entre otras, en el nivel de las subjetividades y el plano de la comunidad local y la nación (Ángel Maya, 1995; Escobar, 2005). Pero estos también pueden devenir en una ampliación del círculo ético, mediante la articulación de la acción colectiva y el discurso político y la negociación por el poder. 
En nuestra perspectiva, acordamos con Amadeo y Caputo (2006) en considerar los espacios participativos convocados a la gestión de políticas públicas como ámbitos de encuentro y negociación de intereses, visiones y poder, donde las relaciones de los participantes crean comunicaciones articuladas por un patrón que resulta de esa relación, en un proceso recursivo.

Con respecto a dichos espacios, Rosenfeld (2005) admite la posibilidad de que, al generarse como nuevo campo con propias reglas de juego a las cuales actores de diferentes campos adhieren, ${ }^{4}$ estos se constituyan en áreas de igualdad que, atravesando el sistema de desigualdades, permitan operar de manera fecunda sobre la realidad; ello se procesará con la conciencia de tener derechos, configurándose una práctica de igualitarismo situada en el campo de lo político ${ }^{5}$.

Esta nueva práctica implica, por lo tanto, la construcción de códigos compartidos derivando en consensos de sentido, o en disensos, con el reconocimiento a un otro legítimo, con posturas propias que pueden coexistir, constituyéndose la comunicación en estos casos- en un proceso emancipador (Alvarado Salgado, 2013).

Lo dicho hasta aquí da cuenta de que los espacios de participación en la implementación de las políticas públicas deberían ser ámbitos de construcción democrática, lejos de los modelos de planificación central que obedecen solo a la lógica de sus autores.

Por ejemplo, en el caso de los planes y programas sociales, para no seguir operando bajo formas asimétricas evidenciadas por la pasividad con la cual se inscriben, son seleccionados y luego beneficiados los destinatarios, sin probabilidad alguna de ejercer conductas proactivas, cuando tal vez sería deseable empoderar a los participantes.

Una vía mediante la cual esta posibilidad lograría concretarse la hallamos en la propuesta de Feijoó, consistente en desarrollar mecanismos de coordinación, no solo entre sectores de efectores de política pública, sino también, y fundamentalmente, incorporando en el diseño de esa política a los destinatarios en su condición de derechohabientes (Feijoó, 2006).

En tanto lugar de encuentro entre organismos estatales y movimientos, asociaciones y organizaciones sociales que representan intereses y expresan demandas de los ciudadanos, los espacios participativos generan un proceso de interacción social entre actores con poder explícito (formal) y los que carecen de él. Ese cambio en los ejercicios de poder implica para el Estado -en las instancias de formulación, definición,

\footnotetext{
${ }^{4}$ Similar al campo de Bourdieu, noción que permite pensar el mundo social no de manera sustancialista, sino como un espacio de relaciones que debe ser entendido en sus diferencias espaciales y temporales y en el cual la participación es producto del aprendizaje social inscripto en el sujeto.

${ }^{5}$ Esta es la idea de Pizzorno (1976) cuando sostiene que al operar en un sistema de intereses (o sea de desigualdades) un sistema de solidaridad sobre la estructura y los valores establecidos, el resultado es un proceso de formación de áreas de igualdad; por lo tanto puede llegar a conformarse un sistema de solidaridad que actúe sobre el sistema de intereses, en la medida en que en un área específica de acción las desigualdades son negadas.
} 
ejecución, seguimiento y evaluación de las políticas públicas- fortalecer la capacidad instituyente (autónoma) de los sujetos involucrados en los objetivos de esa política.

En tal sentido, la apertura de espacios participativos no puede reducirse a otorgar organicidad a la participación, ya que la formalización del espacio no garantiza su sustentación ni su capacidad transformadora; la lógica sectorial corporativa con la que llegan a constituirse ciertos espacios inhibe la incorporación de nuevas visiones, excluye a los grupos no organizados y obstaculiza la generación de proyectos colectivos.

Por ello, la creación y sostenimiento de estos espacios requiere la existencia de actores políticos con voluntad de incidencia, capacidad de negociación y agenda propia.

La institucionalización de la participación ciudadana en la gestión de las políticas públicas es un camino hacia la generación de mayores niveles de democracia, siempre que estos procesos se articulen fuertemente con el sistema político interpelándolo e interactuando con él.

Las prácticas participativas no pueden considerarse como dispositivos meramente técnicos, eficientes o ineficientes, sin vincularlos a cuestiones que atraviesan el orden de lo político, tanto en términos de legitimidad social, derechos y expectativas ciudadanas, como en su vinculación con otros espacios de decisión de la política estatal.

Así, impulsar la participación es abrirse hacia una construcción cultural incierta, habilitando a la población a ser parte de ella.

Los procesos participativos pueden superar las lógicas con las que fueron concebidos: las personas resignifican los espacios, se apropian y generan formas nuevas de decir, hacer y vincularse.

Cabe aclarar que estas consideraciones son formuladas desde nuestro interés en tomar la perspectiva de lo que la política pública ha pensado como espacios participativos.

Si bien no es objeto de este trabajo, no desconocemos la existencia de modos de participación propios de los grupos autoconvocados y autogestionados que pueden ser de signo distintivo frente a los que se producen en espacios participativos en la gestión de políticas públicas.

En general en esos ámbitos participar es paridad frente a la capacidad de generar la norma y frente a la norma misma, capacidad de decidir, mutualidad, etc.; por ello, cuando aquellos grupos deciden participar de llamados del gobierno, traen la lógica de la autoconvocatoria pero a la vez saben que deberán negociar con las lógicas instituidas por los programas en el marco de la política pública.

Por último, pensamos que en el encuadre axiológico que configura la manera democrática de construir ciudadanía en los espacios participativos deben estar presentes el respeto y la justicia. 
El respeto no exclusivamente vinculado a la autoridad y la norma, sino también como reconocimiento a la diferencia, como valoración de fines compartidos que lleva a respuestas solidarias, y la justicia vinculada no solo con el cumplimiento de la norma y la sanción ante las transgresiones, sino, fundamentalmente, referida a criterios de equidad e inclusión (Alvarado Salgado, 2013).

\section{Conclusiones}

El análisis realizado sobre las experiencias concretas nos situó ante proposiciones gubernamentales que definen aquello que consideran la mejor forma de lograr determinados objetivos sociales. Hemos visto que esas proposiciones, resultantes de la interacción -moldeada por marcos institucionales- entre actores del Estado y otros sectores sociales son, en definitiva, ideas que contienen la fuerza y las debilidades de dicha interacción y que, en consecuencia, en su aplicación generarán ciertos efectos sociales, tanto deseados como no deseados.

Formulados desde los niveles centrales, muchos de los programas sociales desarrollados en la región dan cuenta del planteo de una relación asimétrica entre los gestores y los beneficiarios, sin alcanzar los objetivos que inicialmente se habían propuesto y evidenciando en la mayoría de los casos, la contradicción entre su diseño basado en una concepción racional del Estado, que funciona en base a reglas objetivas e impersonales, y su implementación donde se dejan ver fenómenos tales como el clientelismo político (Pérez Rubio y Antequera Durán, 2012).

En síntesis, tanto el recorrido de la literatura consultada, como el análisis de los programas y proyectos con componente participativo nos llevan a considerar que las políticas públicas que se implementan en América Latina y el Caribe muestran la existencia de un escenario de tensión entre una gestión de estas políticas que contemple lo contextualmente situado desde una concepción democrática abierta a la construcción de nuevos significados, y una gestión autoritaria que impone los significados de manera heterónoma.

La democratización de los espacios de participación constituye una estrategia que en el espacio participativo afirma tendencias nuevas $y$, a la vez, un laboratorio para observar la aplicación de las políticas públicas, las metodologías más exitosas y los procesos más consistentes.

En tal sentido, provee de un ámbito de expresión y debate a aquellos sectores sociales cuyas opiniones, anhelos y aspiraciones no encuentran respuesta en las ideas ni en los dispositivos institucionalizados de la democracia representativa. Ello impulsa prácticas de cooperación porque se aprecian las consecuencias de estas estrategias para todos los participantes.

Se trata, sin duda, de un proceso de transformación al promover medios transparentes de información e investigación, de la formación destinada a generar una 
energía social de participación en las comunidades orientada a la vida y decisiones del colectivo social.

Finalmente, y considerando lo desarrollado a lo largo de este artículo, entendemos que uno de los desafíos para las ciencias sociales, como marca Ziccardi (2012), está en la necesidad de consolidar el diálogo entre académicos y quienes desde la gestión pública buscan lograr mejores resultados si se incluye a la ciudadanía en los procesos de toma de decisiones públicas, a la vez que se puede profundizar la construcción de una cultura democrática.

\section{Bibliografía}

Almirón, L., Gómez, L., Ortiz, M., Foio, M., Macarrein O. y Simón R. (2006). Sistematización de los Programas de Desarrollo y Gestión de Recursos Naturales en la población rural de Colonia Carlos Pellegrini, Corrientes, Argentina. En: L. Almirón, H. Altamirano y M. Saavedra, (2011) (Coords.) La ruralidad en Corrientes. Pp. 105-172. Corrientes: Ediciones Moglia.

Alvarado Salgado, S. (2013). De las esperanzas e institucionalidades emergentes. Subjetividades políticas emergentes. En: P. Botero Gómez y A. Palermo (2013) (Coords.) La utopía no está adelante. Generaciones, resistencias e institucionalidades emergentes. Pp. 298-314. Asociación Argentina de Sociología, CINDE y Universidad de Manizales. Buenos Aires: CLACSO.

Álvarez Leguizamón, S. (2009). Una presentación desde América Latina. En: P. Spicker, S. Álvarez Leguizamón y D. Gordon (2009) (Edits.) Pobreza: Un glosario internacional. Pp. 25-37. Buenos Aires: CLACSO-CROP.

Amadeo E. y Caputo S. (2006). Análisis de procesos participativos de diseño e implementación de políticas sociales. Serie Cuadernos de Capacitación. Buenos Aires: Observatorio Social-BID-UNFPA.

Ángel Maya, A. (1995). Desarrollo Sostenible, aproximaciones conceptuales. Quito: Fundación Natura.

Ballart, X. (1992). ¿Cómo evaluar programas y servicios públicos? Aproximación sistemática y estudios de caso. Madrid: Ministerio para las Administraciones Públicas.

Borón, A. (1993). Clases sociales y movimientos sociales en el capitalismo contemporáneo. Margen izquierdo, 8. Buenos Aires: Revista de análisis políticos y culturales, 7-11.

Castoriadis, C. (2006). Poder, política y autonomía [en línea], Traducción de Ignacio de Llorens. Revisión técnica de Fernando Urribarri (última revisión: 2-07-2006), <http://www.socioantro5.blogspot.com.ar/2006/07/poder-poltica-yautonoma.html>, [Consulta: 2 ago. 2013].

Cunill, N. (1991). Participación Ciudadana, dilemas y perspectivas para la democratización de los Estados Latinoamericanos. Caracas: CLAD. 
Escobar, A. (2005). Más allá del tercer mundo: globalización y diferencia, Bogotá: Instituto Colombiano de Antropología e Historia.

Feijoó, M. (2006). Introducción. En: M. Feijoó y M. Poggi (2006) (Coords.) Educación y políticas sociales. Sinergias para la inclusión. Pp. 21-46. Buenos Aires: IIPEUNESCO.

Fleury, S. (2008). Protección social en un mundo globalizado. Cohesión social y desigualdad en América latina y el Caribe, 2008. Buenos Aires, COPPPAL, 10-24.

Foio, M. (2007). El enfoque ecológico de la mente en la corriente sociológica del constructivismo sistémico. XXVI Congreso Asociación Latinoamericana de Sociología, 13-18 agosto, Guadalajara, México (paper).

(2009). Desarrollo sustentable: aplicación de un modelo descentralizado de planificación e intervención territorial. En: J. Mejía Navarrete (2009) (Edit.) Sociedad, cultura y cambio en América Latina. Pp. 203-222. Lima: Universidad Ricardo Palma.

- (2014). Miradas transdisciplinarias en las ciencias sociales en América

Latina. Reflexiones que se articulan con los conceptos de autonomía y alteridad desarrollados por Cornelius Castoriadis. De Prácticas y Discursos [en línea], Cuadernos de Ciencias Sociales, III, 3, <http://depracticasydiscursos.unne.edu.ar/Revista3/pdf/foio.pdf>, [Consulta: 10 mar. 2015].

Foio, M. y Pérez Rubio, A. (2009). El proceso de micro-regionalización como estrategia del desarrollo local: un estudio en la provincia del Chaco. Revista de Estudios Regionales y Mercado de Trabajo, 5, Buenos Aires: CIPSA-SIMEL-CEUR, 265-279. - (2011). Elaboración de un Modelo de Observación de las Políticas Sociales. Aportes de la Teoría Sistémica de Niklas Luhmann, Yuyaykusun II, 4. Lima: Departamento Académico de Humanidades. Universidad Ricardo Palma, 201213.

(2012a). Política neodesarrollista y construcción de subjetividades. El caso argentino. Yuyaykusun II, 5. Lima: Departamento Académico de Humanidades. Universidad Ricardo Palma.

(2012b). Pobreza y políticas públicas. Discursos y prácticas. En: A. Pérez Rubio y N. Antequera Durán (2012) (Comps.) Viejos problemas, nuevas alternativas. Estrategias de lucha contra la pobreza gestadas desde el Sur. Pp. 189-215. Buenos Aires: CLACSO.

García Raggio, A. (2008). Del poder del discurso al discurso del poder. Buenos Aires: EUDEBA.

Heras Monner Sans, A. (2011). En busca de la autonomía: Un análisis sociolinguístico de experiencias asamblearias. Postconvencionales, 3, Caracas: Escuela de Estudios Políticos y Administrativos. Universidad Central de Venezuela, 103-130. 
Heras, A. y Foio, M. (2007). Inclusion and exclusion as semantic field: agent's perspectives through interdisciplinary analysis. En Actas de Second International Conference on Multicultural Discourses. Zhejiang University, 13-15 abril, Hangzhou, China (paper).

Laclau, E. (1994). Poder y representación. Sociedad, 4. Buenos Aires: Facultad de Ciencias Sociales UBA, 5-23.

López Yañez, J. (2003). Abriendo la caja negra. Una perspectiva sistémica sobre el cambio en las organizaciones educativas. XXI, Revista de Educación, 5. Huelva: Universidad de Huelva, 139-155.

- (2005). La ecología social de la organización. Una perspectiva educativa. Madrid: La Muralla.

Luhmann, N. (2006). La sociedad de la sociedad, México: Herder: Universidad Iberoamericana.

Matellanes, M. (1999). Capitalismo Siglo XXI, la impostergable alternativa: imperio hobbesiano o multitud spinozista. Sociedad, 5. Buenos Aires: Facultad de Ciencias Sociales UBA.

Pérez Rubio, A. y Foio, M. (2009). El discurso de la participación y los procesos de desarrollo local. En: A. Heras y D. Burin (2009) (Comps.) Trabajo, Desarrollo, Diversidad. Pp. 81-93. Buenos Aires: Ciccus-Incluir.

Pérez Rubio A. y Antequera Durán, N. (2012). Introducción. En: A. Pérez Rubio y N. Antequera Durán (2012) (Comps.) Viejos problemas, nuevas alternativas. Estrategias de lucha contra la pobreza gestadas desde el Sur. Pp. 12-19. Buenos Aires: CLACSO.

Pintos, J. (1994). Sociocibernética: marco sistémico y esquema conceptual. En: J. Delgado y J. Gutiérrez (1994) (Edits.) Métodos y técnicas cualitativas de investigación social. Pp. 563-580. Madrid: Síntesis.

Piñango, Ramón (2003). Políticas públicas para orientar el rumbo de un país. En J. Kelly, (2003) (Coord.) Políticas públicas en América Latina. Teoría y práctica. Pp. 249277. Caracas: Ediciones IESA.

Pizzorno, Alessandro (1976). Introducción al Estudio de la Participación Política, SIAP, Buenos Aires: Planteos.

Repetto, F. (2001). Gestión pública y desarrollo social en los noventa: Las trayectorias de Argentina y Chile. Buenos Aires: Prometeo.

(2005). La dimensión política de la coordinación de programas y políticas sociales: una aproximación teórica y algunas referencias prácticas en América Latina. En F. Repetto (2005) (Edit.) La gerencia social ante los nuevos retos del desarrollo en América Latina, INDES. Pp. 39-98. Guatemala: Magna Terra Editores.

Rivas, R. (2013) Presente y futuro de la democracia latinoamericana. Cuestiones de Sociología, 9 [en línea], disponible en: 
http://www.cuestionessociologia.fahce.unlp.edu.ar/article/view/CSn09a38/4580 [Consulta: 15 may 2014].

Rosenfeld, M. (2005). Dilemas de la participación social: El encuentro entre las políticas públicas y la sociedad civil. Observatorio Social. Cuaderno, $N^{\circ} 7$, Dilemas Actuales de la Participación Social, septiembre 2005 [en línea], disponible en: www.observatoriosocial.com.ar/images/pdf_cuadernillos/cuader7.pdf [Consulta: 20 nov 2014].

Sader, E. (2012). El estado de las ciencias sociales en América Latina y el Caribe. Crítica y Emancipación, 9, primer semestre 2013. Buenos Aires: CLACSO, 11-21.

Subirats, J. (1989). Análisis de Políticas Públicas y Eficacia de la Administración. Madrid: Imprenta Nacional del Boletín del Estado.

Spicker, P., Álvarez Leguizamón, S. y Gordon, D., (Edits.) (2009). Pobreza: Un glosario internacional. Buenos Aires: CLACSO-CROP.

Toer, M., Martínez Sameck, P., Agilda, L., Barassi, S., Burbano de Lara, A., Garrido, N., Goldstein, A., Melendi, L., Montero, F. y Salas Oroño, A. (2011). Reflexiones sobre algunas cuestiones de la actual coyuntura latinoamericana. En: Actas del Congreso de Pensamiento Político Latinoamericano. Buenos Aires: Universidad Popular Madres de Plaza de Mayo.

Verón, E. (1969). Conducta, estructura y comunicación, Buenos Aires: Jorge Álvarez.

Ziccardi, A. (2012). Sobre la participación ciudadana en las políticas públicas del ámbito local. En: M. Canto Chac (2012) (Comp.) Participación ciudadana en las políticas públicas. Pp. 205-221. México: Siglo XXI edit. 